\title{
Teste das pirâmides coloridas de Pfister e a criatividade em crianças
}

\author{
Anna Elisa de Villemor-Amaral' \\ Universidade São Francisco, Itatiba - SP - Brasil \\ Raquel Rossi Tavella \\ Centro Universitário Nossa Senhora do Patrocínio, Itu - SP - Brasil \\ Lucila Moraes Cardoso \\ Universidade Estadual do Ceará, Fortaleza - CE - Brasil \\ Fabíola Cristina Biasi \\ Pâmela Malio Pardini Pavan \\ Universidade São Francisco, Itatiba - SP - Brasil
}

\begin{abstract}
Resumo: O objetivo deste trabalho foi verificar evidências de validade para o uso do teste das pirâmides coloridas de Pfister com crianças, especificamente no que diz respeito à criatividade. Participaram do estudo 56 crianças, de ambos os sexos, que cursam o sexto ano do ensino fundamental de escolas públicas localizadas em uma cidade do interior de São Paulo. A amostra foi dividida em dois grupos extremos com base nos resultados do teste de criatividade figural infantil. Posteriormente, foi aplicado o teste das pirâmides coloridas de Pfister. As crianças mais criativas apresentaram no Teste de Pfister aumento de síndrome de estímulo, resultado que pode auxiliar a identificar as crianças que possuem maior energia, abertura para os estímulos e interesse para produzir, o que condiz com a literatura. Afinal, pessoas criativas tendem a ter mais necessidade de se expressar.
\end{abstract}

Palavras-chave: avaliação psicológica; criatividade; teste das pirâmides coloridas; validade do teste; desenvolvimento infantil.

\section{PFISTER'S COLORED PYRAMIDS TEST AND CREATIVITY IN CHILDREN}

\begin{abstract}
The aim of this work was to verify evidence of validity for the use of Pfister's colored pyramids test with children, specifically with regard to creativity. Participated in this study 56 children of both genders, who attend the sixth grade of elementary school from public schools, located in an inner city of São Paulo State. The sample was divided into two extreme groups on the basis of the results of the infant figural creativity test. Later it was applied Pfister's Colored Pyramids Test. The most creative children showed that Pfister's test increase in stimulus syndrome. This result can help to identify children who have more energy, openness and interest to produce, which matches the literature. After all, creative people tend to have more need to express themselves.
\end{abstract}

Keywords: psychological assessment; creativity; colored pyramid test; test validity; childhood development.

1 Endereço para correspondência: Anna Elisa de Villemor-Amaral, Rua Alexandre Rodrigues Barbosa, 45, Centro - Itatiba - SP - Brasil. CEP: 13251-900. E-mail: anna.villemor@saofrancisco.edu.br. 
TESTE DE LAS PIRÁMIDES COLORIDAS DE PFISTER Y LA CREATIVIDAD EN LOS NINÕS

\begin{abstract}
Resumen: El objetivo de este trabajo fue verificar las evidencias de validez para el uso del test de las pirámides coloridas de Pfister con los niños, en particular en lo que respecta a la creatividad. Participaron del estudio 56 niños de ambos sexos que asisten al sexto grado de enseñanza fundamental de escuelas públicas situadas en una ciudad en el interior de São Paulo. La muestra se dividió en dos grupos extremos con base en los resultados del test de creatividad figural infantil. Más tarde se utilizó el test de las pirámides coloridas de Pfister. Los niños más creativos mostraron aumento el test de Pfister en el síndrome de estímulo, y este resultado puede ayudar a identificar a los niños que tienen mayor interés para producir, o que es coherente con lo que dice la literatura. Después de todo, las personas creativas tienden a tener más necesidad de expresarse.
\end{abstract}

Palabras clave: evaluación psicológica; creatividad; test de las pirámides coloridas; validacion de test; desarrollo infantil.

O teste das pirâmides coloridas de Pfister (TPC) é um método expressivo que possibilita a avaliação dos aspectos emocionais e cognitivos da personalidade do indivíduo, por meio da maneira como este dispõe quadrículos coloridos sobre esquemas de pirâmides. O TPC é um instrumento não verbal de fácil manejo e aceitação e propício para a população infantil (Villemor-Amaral, 2014).

Esse teste foi criado, na década de 1940, por Max Pfister, que se baseou em sua experiência com as cores e formas desde a época em que era arquiteto, bailarino e cenógrafo. Essas habilidades técnicas e sua sensibilidade artística levaram-no a observar a relevância da experiência subjetiva que as cores produziam nas pessoas. As cores são estímulos naturais comuns à vida da maioria das pessoas, e diversas culturas as utilizam em suas manifestações artísticas e religiosas, com imputações simbólicas comuns que se repetem ao longo dos anos, com poucas variações em diferentes grupos culturais (Villemor-Amaral, 2012).

Os estudos sobre o uso do TPC com a população infantil ainda são escassos. Encontrou-se a pesquisa de Jacob, Loureiro, Marturano, Linhares e Machado (1999), que tiveram como objetivo demonstrar o funcionamento afetivo de 50 crianças com idade entre 8 e 12 anos, de ambos os sexos, com base nos testes desenho da casa-árvore-pessoa (house-tree-person - HTP) e TPC. Os sujeitos foram divididos em dois grupos, o primeiro com 25 sujeitos com atraso escolar, e o segundo grupo apresentando desempenho escolar satisfatório e idade compatível com a série cursada. Os resultados indicaram que as crianças sem atraso escolar utilizam melhor os recursos intelectuais e afetivos, e possuem um nível de exigência elevado, corroborando a compreensão do que têm vivenciado nessa etapa do desenvolvimento.

Cardoso e Capitão (2007) utilizaram o TPC para verificar se existem diferenças na estruturação cognitiva e aspectos afetivos de crianças com surdez. A amostra contou com a participação de 81 crianças com surdez que foram comparadas com 37 crianças ouvintes, de idades entre 6 a 12 anos. As crianças com surdez não tiveram dificuldades em relação às instruções de montagem das pirâmides. Os resultados indicaram que as crianças ouvintes têm um nível intelectual mais sofisticado quando comparadas 
às crianças com surdez, que, por sua vez, apresentaram mais ansiedade que as crianças ouvintes.

Cardoso e Capitão (2012) realizaram uma pesquisa com o objetivo de correlacionar o TPC com o desenho da figura humana (DFH)-Koppitz e o DFH-Sisto. Participaram do estudo 118 crianças de ambos os sexos, com idades entre 6 e 12 anos. Os resultados apresentaram 24 correlações significativas entre os indicadores do TPC com o DFH, revelando que os indicadores de ambos os instrumentos avaliam traços de personalidade semelhantes.

No estudo de Villemor-Amaral, Pardini, Tavella, Biasi e Migoranci (2012), foi comparado o desenvolvimento cognitivo e emocional de 85 crianças de 6 e 12 anos, de ambos os sexos, no TPC. Os resultados indicaram um aumento significativo da cor amarela, da síndrome de estímulo e de tapetes puros e com início de ordem nas crianças de 6 anos. Já as crianças de 12 anos tiveram aumento significativo nas cores branca e preta e na estrutura em manto. O instrumento se mostrou sensível para identificar variação emocional com base nas teorias do desenvolvimento que afirmam que crianças de 6 anos apresentam maior imaturidade quando comparadas com as de 12 anos, que teriam maior capacidade de lidar com as emoções e controle de sentimentos e impulsos.

Quando Pfister idealizou o TPC, considerou que o interesse e a escolha das cores estavam diretamente relacionados com a dinâmica emocional do indivíduo e verificou, por exemplo, que a escolha das cores poderia estar associada a emoções mais excitadas quando o indivíduo seleciona cores quentes ou mais calmas quando escolhe as cores frias (Villemor-Amaral \& Franco, 2010). No mesmo sentido, ao analisar os desenhos coloridos, Hammer (1989) observou certa universalidade no fato de que sujeitos psicologicamente sadios utilizam as cores quentes com mais segurança, enquanto sujeitos mais inibidos e constritos usam mais preto, marrom e azul.

Essas observações demonstram a possibilidade de usar as cores para conhecer outros aspectos do funcionamento psíquico que estejam atrelados à dinâmica afetiva de uma pessoa, tornando possível inferir a maneira como ela lida com diferentes situações da vida. A criatividade, por exemplo, é um construto que aparece na literatura frequentemente ligada à dinâmica emocional. Para Winnicott (1975), a afetividade e a liberdade de expressão são fatores fundamentais na manifestação da criatividade. Sakamoto (2012) menciona que Winnicott e Csikszentmihalyi, ao descreverem a criatividade, consideraram que as relações afetivas e o ambiente, desde o início da vida, permitem o desenvolvimento do sentimento de segurança, elemento considerado fundamental para o aparecimento da criatividade. Assim, para o autor, a afetividade permeia a criatividade e possibilita a identificação de ambientes, pessoas e processos que se apresentam de modo mais criativo que outros (Sakamoto, 2012). Associado a isso, Alencar e Fleith (2003) afirmam que os fatores de ordem sociocultural, ou seja, valores e regras sociais, podem contribuir de forma expressiva para a manifestação da criatividade ou, pelo contrário, impedir e reprimir esse potencial. 
Como apontam diversos estudos as pessoas mais criativas são mais bem caracterizadas como aquelas amplamente interessadas, ambiciosas, autoconfiantes, com tendência à não conformidade e que se esforçam continuamente para experimentar algo novo (Fink \& Woschnjak, 2011; Kaufman, Pumaccahua, \& Holt, 2013). Assim, pode-se dizer que "de especial relevância para a expressão da criatividade é a disposição para correr riscos e aprender com os próprios erros. A coragem é, pois, indubitavelmente um atributo de fundamental importância, uma vez que a criatividade implica lidar com o desconhecido" (Alencar, 1998, p. 20).

Associado a isso, Wechsler (2004) trata da importância da motivação para criar. Nessa mesma perspectiva, Torrance (1970) afirma que a criatividade pode favorecer a sensibilidade das pessoas e interferir no modo de identificar e solucionar problemas.

Wechsler $(1998$, p. 91) compreende que a criatividade deve ser entendida dentro de um modelo multidimensional, pois é composta por elementos cognitivos e emocionais que são resultado da interação entre "processos cognitivos, ambiente, características da personalidade e elementos inconscientes". A autora ressalta que o modelo multidimensional é adequado para entender a criatividade, pois não define um único fator para ser aplicado em todas as situações e que possa explicar todas as formas de manifestação criativa; assim, não é apropriado falar sobre uma única criatividade e sim de criatividades, no plural. Desse modo, de acordo com Romo (2008), todo indivíduo é dotado de habilidades criativas que podem se apresentar em maior ou menor grau e, sendo assim, não pode ser considerado criativo em todas as áreas. Cada um possui talentos que poderão proporcionar a manifestação da criatividade em uma área específica.

Nessa perspectiva de que a criatividade é multidimensional, Nakano, Wechsler e Primi (2011) criaram o teste de criatividade figural infantil (TCFI), que visa fornecer uma medida quantitativa geral do nível de criatividade. Para o estudo de evidências de validade, participaram 120 crianças do interior do Estado de São Paulo com idade entre 7 e 10 anos. Todas cursavam entre o $1^{\circ}$ e o $5^{\circ}$ ano do ensino fundamental na mesma instituição de ensino. Foram aplicados o TCFI e o teste figural de Torrance. O estudo apresentou bons resultados no que se refere à existência de validade concorrente entre os instrumentos, na medida em que os quatro fatores avaliados pelo TCFI e dois índices criativos do teste de Torrance mostram-se altamente significativos $(r=0,81$ a 0,$94 ; p<0,001)$.

No que se refere ao estudo de precisão do TCFI, utilizou-se o método do teste-reteste. Para tal, 30 crianças, de ambos os sexos, escolhidas aleatoriamente, responderam ao TCFI duas vezes. Houve um intervalo de 30 dias entre as duas aplicações. Por meio da correlação de Pearson, verificou-se alto índice de precisão, com elevada relação entre o teste e reteste entre todos os fatores, sendo $r=0,981$ para o fator $1, r=0,903$ para o fator $2, r=0,952$ para o fator 3 e $r=0,917$ para o fator 4 .

Alencar e Fleith (2003) asseguram que a criatividade é composta por aspectos de natureza intrapsíquica e de ordem sociocultural. A partir da hipótese de que o construto 
criatividade tem relação com a dinâmica emocional das pessoas, Villemor-Amaral e Franco (2010) realizaram um estudo com adultos, artistas plásticos bem-sucedidos, e verificaram que os artistas faziam pirâmides com alto grau de elaboração e que foram consideradas raras na amostra normativa, tais como mosaicos e estruturas assimétricas dinâmicas, demonstrando sua criatividade e originalidade na maneira de lidar com o estímulo colorido e a estrutura da pirâmide.

Considerando essa possível relação entre criatividade e personalidade, tem-se a hipótese de que o TPC seria capaz de identificar a manifestação criativa em crianças, seja por meio do uso das cores mais estimulantes, seja por meio de aspectos formais mais elaborados. Sendo assim, este trabalho tem como objetivo verificar novas evidências de validade para o uso do TPC com crianças, especificamente no que diz respeito à criatividade.

\section{Método}

\section{Participantes}

Participaram da primeira etapa do estudo 90 crianças, com 11 e 12 anos, de ambos os sexos, que cursam o sexto ano do ensino fundamental em escolas públicas localizadas em uma cidade do interior de São Paulo. Para a segunda etapa, selecionaram-se 56 crianças que apresentaram resultados extremos no TCFI, considerando as normas do teste por série e tipo de escola.

O grupo de crianças menos criativas foi formado por 26 indivíduos, sendo 19 com 11 anos e sete com 12 anos. Nesse grupo, 14 dos participantes eram do gênero masculino. Como critério de inclusão, utilizaram-se, nesse grupo, os protocolos do TCFI (Nakano et al., 2011), classificados como abaixo da média (percentil entre 20 e 34) e inferior (percentil abaixo de 20), com base nas tabelas de série e escola, o que, para os autores, sugeria a necessidade do desenvolvimento de características criativas.

O grupo de crianças mais criativas foi formado por 30 indivíduos: 29 com 11 anos e um com 12 anos. Nesse grupo, dez indivíduos eram do gênero masculino. Esse grupo foi classificado, pelo manual do TCFI, com o percentil superior (acima de 81) e acima da média (entre 65 e 80), sugerindo indivíduos que possuem alto nível de criatividade.

\section{Instrumentos}

Teste das pirâmides coloridas de Pfister (TPC)

O TPC (Villemor-Amaral, 2012) é composto por um jogo de três cartões em papel bege, com o desenho de uma pirâmide e um jogo de quadrículos coloridos, constituídas por dez cores, subdivididas em 24 tonalidades. Para realização do teste, o examinando é convidado a construir pirâmides, uma de cada vez, até totalizar três pirâmides, e deve fazê-las de modo que fiquem bonitas do seu ponto de vista. Em seguida, é feito um inquérito em que se indaga sobre qual dentre as três pirâmides feitas seria a que mais lhe agrada e a que menos Ihe agrada. Estima-se que a aplicação do TPC dure cerca de 20 minutos. 
Para análise do TPC, primeiramente as respostas são codificadas, considerando a frequência das cores, as combinações no uso das cores, a sequência em que as cores foram dispostas e a estabilidade no uso das cores escolhidas. Na análise da frequência das cores, é preciso considerar a porcentagem em que o examinando usou cada uma delas e compará-las com um padrão normativo.

Para analisar as combinações das cores, é preciso verificar se o examinando combinou o uso de algumas cores predeterminadas no manual. Esses dados compõem os indicadores denominados cores por dupla, quando duas combinações predeterminadas são identificadas, e síndromes cromáticas, quando mais de duas cores preestabelecidas são visualizadas no protocolo do examinando.

No que se refere ao aspecto formal, destaca-se que esse indicador se relaciona com o modo como as cores são dispostas no esquema de pirâmide, de modo que, ao final da colocação de todas as cores, tem-se uma configuração da forma. E, por fim, a estabilidade na escolha das cores é considerada no indicador fórmula cromática, que considera a quantidade de cores usadas em uma única pirâmide, em duas pirâmides e nas três pirâmides.

Teste de criatividade figural infantil (TCFI)

Pautando-se no modelo multidimensional da criatividade proposto por Torrance, foi criado o TCFI (Nakano et al., 2011), que é uma avaliação quantitativa do nível de criatividade e das características criativas das crianças, indicando aquelas que necessitam ser estimuladas. O TCFI avalia 12 características que compõem a criatividade: fluência, flexibilidade, elaboração, originalidade, expressão de emoção, fantasia, movimento, perspectiva incomum, perspectiva interna, uso de contexto, extensão de limites e títulos expressivos. Essas características são combinadas entre si, compondo quatro fatores, que são: 1. enriquecimento de ideias, 2. emotividade, 3. preparação criativa e 4. aspectos cognitivos. Além disso, o teste fornece uma pontuação total de criatividade, que é denominada de fator geral.

No TCFI, a criança deve fazer três atividades que envolvem desenho. Na primeira, ela terá cinco minutos para imaginar com que se parece um estímulo não definido e completar a figura; na segunda tarefa, terá dez minutos para imaginar com que se parecem outros dez estímulos e completá-los; e na última, a criança deverá criar o maior número possível de desenhos a partir de um mesmo estímulo, que é repetido 30 vezes num tempo máximo de dez minutos. A todos os desenhos, a criança deverá atribuir um título durante a realização da tarefa. O tempo total de administração é de aproximadamente 40 minutos.

\section{Procedimentos}

Primeiramente, o projeto foi submetido a um Comitê de Ética em Pesquisa da Universidade São Francisco (protocolo Certificado de Apresentação para Apreciação 
Ética (Caae) n. 0229.0.142.000-11). Após aprovação, foram estabelecidas parcerias com escolas públicas localizadas em uma cidade do interior de São Paulo. A direção das escolas encaminhava os Termos de Consentimento Livre e Esclarecido (TCLE) aos pais. Após a confirmação do número de crianças autorizadas, foram agendadas as datas para administração do instrumento.

O TCFI foi administrado de forma coletiva em sessão de aproximadamente 40 minutos. Após análise dos resultados, realizou-se a seleção de crianças para compor os grupos extremos. No dia seguinte, foi administrado o TPC nas crianças selecionadas. A administração ocorreu em sessão individual de aproximadamente 20 minutos. Em seguida, os protocolos do TPC foram inseridos num programa próprio (Miguel \& Villemor-Amaral, 2006) e depois exportados para o Statistical Package for Social Science (SPSS) para realizar as análises estatísticas.

\section{Resultados}

Fizeram-se os testes $t$ de Student e $d$ de Cohen para comparar a frequência de cada cor entre os dois grupos. A única cor que apresentou diferença significativa do ponto de vista estatístico foi o laranja ( $t=-2,02, p=0,04, d=0,55)$, verificando o aumento dessa cor nas crianças mais criativas. Em seguida, as mesmas análises foram feitas comparando os dois grupos em relação às síndromes cromáticas (Tabela 1).

\section{Tabela I. Comparação das médias das síndromes cromáticas entre os grupos com diferentes níveis de criatividade}

\begin{tabular}{llccccc}
\hline Síndromes cromáticas & Grupos & Média & Desvio padrão & $t$ & $d$ & $p$ \\
\hline Estímulo & Menos criativas & 28,53 & 11,59 & & & \\
& Mais criativas & 36,29 & 13,86 & $-2,22$ & 0,60 & 0,03 \\
\hline Incolor & Menos criativas & 17,77 & 9,88 & & & \\
& Mais criativas & 10,96 & 7,95 & & 0,77 & 0,01 \\
\hline
\end{tabular}

Fonte: Elaborada pelas autoras.

A Tabela 1 apresenta os resultados significativos na comparação das síndromes cromáticas entre os dois grupos, em que se utilizou o teste $t$ de Student com um nível de significância de $p<0,05$. Pode-se observar que as crianças mais criativas apresentaram mais síndrome de estímulo $(t=-2,22, p=0,60, d=0,03)$, e as crianças menos criativas apresentaram mais síndrome incolor $(t=2,84, p=0,77, d=0,01)$ que as mais criativas.

Após a comparação da média de uso das cores e das síndromes cromáticas, foi feita a comparação da frequência do aspecto formal e fórmula cromática do TPC entre os dois grupos, por meio do qui-quadrado. Não houve diferença entre os grupos em relação ao aspecto formal, e, no que se refere à fórmula cromática, a ampla e 
flexível apresentou diferença significativa entre os dois grupos $\left(x^{2}=6,330, p=0,001\right)$, e o grupo de crianças menos criativas teve média aumentada em comparação ao grupo mais criativo.

\section{Discussão e conclusão}

Nos resultados, observou-se o aumento do laranja no grupo mais criativo. O laranja é interpretado como a cor relacionada ao desejo de ser reconhecido, de se fazer valer pela produtividade (Villemor-Amaral, 2012, 2014), corroborando a visão de Alencar (1998), Alencar e Fleith (2003), Fink e Woschnjak (2011) e Kaufman et al. (2013) de que a motivação é um aspecto importante para a manifestação da criatividade. Para Wechsler (2004), a motivação para criar pode ser compreendida como resultado da interação entre afetividade, cognição e ação criativa.

Os valores mais baixos do laranja que esteve associado ao grupo de crianças menos criativas no TCFI podem revelar, segundo Villemor-Amaral $(2012,2014)$, inibição e passividade, ou seja, características que contribuem de forma significativa para a contenção da criatividade. Esse dado encontra apoio na visão de Winnicott (1975), quando esse autor defende que as relações afetivas permitem que o indivíduo desenvolva o sentimento de segurança e que essa seria uma condição considerada essencial para a expressão criativa. Também Hammer (1989) observou a diminuição de cores quentes nos desenhos de pessoas inibidas e inseguras. Esse resultado reforça a importância de um ambiente que favoreça a manifestação da criatividade, conforme apontado por Sakamoto (2012) e Alencar e Fleith (2003).

No que se refere aos dados da Tabela 1, verificou-se aumento da síndrome de estímulo nas crianças mais criativas. Essa síndrome é resultado da soma das cores vermeIha, amarela e laranja, isto é, das cores estimulantes ou quentes, indicando capacidade de extroversão e contato afetivo social. Já a síndrome incolor, que apareceu aumentada no grupo de crianças consideradas menos criativas, é formada pela frequência das cores preta, branca e cinza, indicando negação, repressão de estímulos e ainda fuga de situações afetivas (Villemor-Amaral, 2012, 2014). Esse dado novamente corrobora a ideia de que as pessoas mais criativas são mais facilmente estimuladas por vivências afetivas do que as pessoas menos criativas (Alencar \& Fleith, 2003; Torrance, 1970; Wechsler, 2004).

A ausência de diferença significativa na comparação do aspecto formal foi o contrário do esperado a partir do estudo de Villemor-Amaral e Franco (2010), no qual o grupo de artistas plásticos teve presença marcante de estrutura em mosaico e estrutura assimétrica dinâmica. O fato de crianças de 11 e 12 anos não terem apresentado resultados significativos nesses aspectos formais do teste evidencia que nem mesmo as crianças mais criativas têm como, nessa faixa etária, expressar sua criatividade de modo semelhante ao adulto, uma vez que ainda não apresentam recursos intelectuais e afetivos suficientemente amadurecidos, condição necessária para a execução de estruturas. Trata-se, portanto, de uma consequência da imaturidade comum nessa faixa etária, mesmo em crianças com desempenho mais criativo no TCFI. 
O aumento da fórmula ampla e flexível no grupo menos criativo também não era esperado, uma vez que esse indicador se relaciona classicamente a uma capacidade de realização mais enérgica e, possivelmente, mais direcionada para objetivos definidos, denotando capacidade de ajustamento em função da flexibilidade e certa maturidade (Villemor-Amaral, 2012, 2014). Porém, como não apareceu uma fórmula cromática dominante para crianças mais criativas, o entendimento desse achado fica comprometido, sendo necessários mais estudos que ajudem a compreender tal resultado.

É importante ressaltar que, conforme já mencionado, de acordo com Wechsler (1998), a criatividade deve ser compreendida como um modelo multidimensional, isto é, existem diversos tipos de criatividade. Cada indivíduo possui talentos específicos que poderão levar a manifestações criativas em situações variadas e que também podem ser expressas de modo distinto (Nakano et al., 2011). Nesse modelo multidimensional da criatividade, enfatiza-se que o TCFI mede apenas a criatividade figural, não descartando a possibilidade de o indivíduo ser criativo em outros aspectos.

O conjunto dos dados sugere que o TPC, por meio do laranja e da síndrome de estímulo em maior quantidade nas crianças mais criativas e da síndrome incolor em maior quantidade nas crianças menos criativas, mostra a contribuição desse teste na compreensão a respeito da facilidade para ser mais criativo e ter maior interesse em produzir, o que está condizente com a literatura que afirma que pessoas criativas tendem a ter mais necessidade de se expressar e são persistentes, facilitando a produtividade e favorecendo a aprendizagem com os próprios erros.

Assim, o presente estudo corrobora os demais artigos sobre o TPC (Jacob et al., 1999; Cardoso \& Capitão, 2007, 2012; Villemor-Amaral et al., 2012) na medida em que integra esforços em relação às evidências de validade do instrumento. É importante, contudo, destacar que esse conjunto de dados não pode ser considerado suficiente para indicar o TPC como um instrumento para avaliação da criatividade, uma vez que esse construto é multideterminado. Além disso, o presente estudo contou com um número limitado de participantes, e, certamente, estudos com amostras maiores e outros critérios para avaliar a criatividade podem elucidar melhor como o TPC reflete a capacidade criativa em crianças.

\section{Referências}

Alencar, E. M. L. S. (1998). Promovendo um ambiente favorável à criatividade nas organizações. Revista de Administração de Empresas São Paulo, 38(2), 18-25.

Alencar, E. M. L. S., \& Fleith, D. S. (2003). Barreiras à criatividade pessoal entre professores de distintos níveis de ensino. Psicologia: Reflexão e Crítica, 16(1), 63-69.

Cardoso, L. M., \& Capitão, C. G. (2007). Avaliação psicológica de crianças surdas pelo Teste das Pirâmides Coloridas de Pfister. Psico-USF, 12(2), 135-144. 
Cardoso, L. M., \& Capitão, C. G. (2012). Evidence of incremental validity between Pfister test and human figure drawing. International Journal of Psychology and Behavioral Sciences, 2, 120-129.

Fink, A. \& Woschnjak, S. (2011). Creativity and personality in professional dancers. Personality and Individual Differences, 51, 754-758.

Hammer, E. (1989). A interpretação clínica dos desenhos projetivos. São Paulo: Casa do Psicólogo.

Jacob, A. V., Loureiro, S. R., Marturano, E. M., Linhares, M. B. M., \& Machado, V. L. S. (1999). Aspectos afetivos e o desempenho acadêmico de escolares. Psicologia Teoria e Pesquisa, 15(2), 153-162.

Kaufman, J. C., Pumaccahua, T. T., \& Holt, R. E. (2013). Personality and creativity in realistic, investigative, artistic, social, and enterprising college majors. Personality and Individual Differences, 54, 913-917.

Miguel, F. K., \& Villemor-Amaral, A. E. (2006). Correção do Teste das Pirâmides Coloridas de Pfister. Patente: Programa de Computador. Número do registro: BR. Patente n. 076123. Rio de Janeiro: Instituto Nacional da Propriedade Industrial.

Nakano, T. C., Wechsler, S. M., \& Primi, R. (2011). Teste de criatividade figural infantil. São Paulo: Vetor.

Romo, M. (2008). Creatividad em los domínios artístico y científico y sus correlatos educativos. In M. F. Morais \& S. Bahia (Orgs.). Criatividade: conceitos, necessidades e intervenção (pp. 66-90). Braga: Psiquilíbrios.

Sakamoto, C. K. (2012). Criatividade e a construção da realidade contemporânea. Revista Trama Interdisciplinar, 3(1), 86-96.

Torrance, E. P. (1970). Encouraging creativity in the classroom. lowa: Brown Company Publishers.

Villemor-Amaral, A. E. (2012). As pirâmides coloridas de Pfister. São Paulo: Casa do Psicólogo.

Villemor-Amaral, A. E. (2014). As pirâmides coloridas de Pfister - versão para crianças e adolescentes. São Paulo: Casa do Psicólogo.

Villemor-Amaral, A. E., \& Franco, R. R. C. (2010). A psicopatologia fenômeno-estrutural e o teste das pirâmides coloridas de Pfister. In A. E. Villemor-Amaral \& L. Yazigi (Orgs.). Psicopatologia fenômeno-estrutural (pp. 167-184). São Paulo: Casa do Psicólogo.

Villemor-Amaral, A. E., Pardini, P. M., Tavella, R. R., Biasi, F. C., \& Migoranci, P. B. (2012). Evidências de validade do teste de Pfister para avaliação de crianças. Avaliação Psicológica, 11(3), 423-434.

Wechsler, S. M. (1998). Criatividade: descobrindo e encorajando. São Paulo: Psy. 
Wechsler, S. M. (2004). Avaliação da criatividade por figuras e palavras: testes de Torrance - versão brasileira (2a ed. revisada). Campinas: Lamp, Impressão Digital do Brasil.

Winnicott, D. W. (1975). O brincar e a realidade. Rio de Janeiro: Imago.

Submissão: 10.6.2013

Aceitação 20.8.2014 